\title{
Detection of reckless driving in the Sub-Saharan informal public transportation system using acceleration-sensing telematics
}

\author{
Justin M. Schietekat and Marthinus J. Booysen \\ Department of Electrical and Electronic Engineering, Stellenbosch University \\ Stellenbosch, South Africa. \\ mjbooysen@sun.ac.za
}

\begin{abstract}
Many lives are lost annually in Sub-Saharan Africa's unique and vibrant informal public transportation system due to accidents caused by reckless driving, fatigue, and illegal overloading. Fleet management has been used extensively to determine the location of mobile assets in the event of theft, or as part of operational management. One of the main objectives of the work presented in this paper, is to build on existing fleet management systems to improve safety and drastically reduce road accidents and the unnecessary loss of lives. Through a thorough literature survey it was concluded that existing work on vehicle tracking and reporting systems, do not use accelerometers to monitor or detect reckless driving, despite the obvious advantages. Similar to other tracking devices, this project uses global positioning to determine position and velocity, but this project also incorporates the use of a 3-axis accelerometer augmented by a theoretical behavioral model to detect and report reckless behavior to a central online server. The accelerometer results show a clear difference between safe and reckless driving.
\end{abstract}

Index Terms-Informal public transport, Intelligent Transport Systems, Accelerometers in Telematics.

\section{INTRODUCTION}

Fleet monitoring is a technique used to obtain various measurable data from any type of vehicle. The system depends on, and takes advantage of the fact that there are wireless data communication networks available practically in all the areas where vehicles operate. Such a system only reports small packets of vital information which does not require a high bandwidth communication channel. Telematics typically describes any integrated use of telecommunications and informatics, also known as Information and Communications Technology (ICT). Telemetry is the transmission of measurements from the location of origin to the location of computing and consumption, without affecting the control on the remote subject.

Vehicle telematics has various practical applications and are used in numerous fields in the business and private sector. In the private sector it is used to monitor the location, speed, status and behavior among other features of personal vehicles and fleets. These uses move to the commercial sector when third-party companies are involved to supply the device and a recovery service, if a vehicle was stolen. The business sector's uses include those of the private sector but may include features specifically for fleet management such as, driver identification, driver ratings, on-going logging, reminder listings, fuel profiling, warning systems for when vehicles leave usual operating areas, vehicle immobilization, vehicle status such as temperature, fuel levels, oil pressure etc.

The work in [1] visually demonstrate the unique nature of the public transportation industry in Sub-Saharan Africa (SSA), and the reader is encouraged to peruse these for a deeper understanding of the context of this work. To allow for the monitor and control of this industry, additional sensing is required to monitor driver behavior, which will allow government agencies to save lives.

\section{A. Contribution}

This work aims to monitor and report, in addition to the usual location and speed, also the behavior of the driver. The primary objectives for this work is the actively monitoring of a vehicle's location, velocity and driver behavior whilst transmitting relevant data to a centralized web server. Data such as acceleration, speed and tilt angle of the vehicle was measured and used to characterize driver behavior.

The rest of the paper is organized as follows: The related work section covers numerous examples of similar vehicle telematic systems that are being developed or prepared around the world. The next two sections explain the system design and describe the results of the performed tests, which show how to distinguish safe from reckless braking and turning. The conclusion summarizes the paper.

\section{RELATED WORK}

This literature study conveys information concerning the necessity for vehicle telematics in the transport infrastructures of Sub-Saharan Africa and the status of current vehicle telematics around the world. eCall in Europe is one example of a government project that is helping to further vehicle telematics. The European Union's goal is to have every vehicle equipped with the eCall system by 2015 [3]. The eCall system is embedded within a vehicle and linked to the vehicle's air 


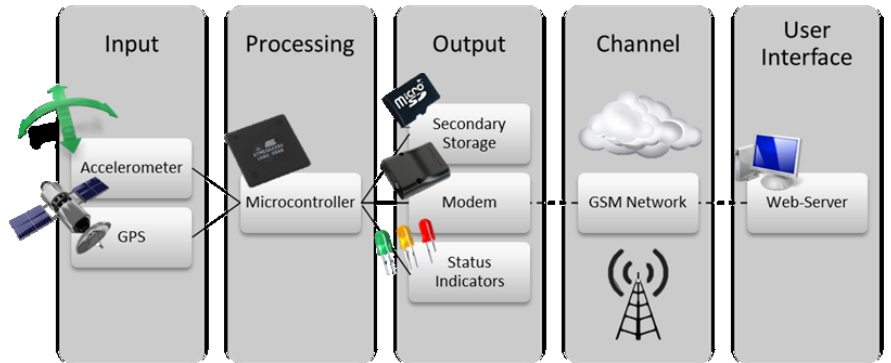

Fig. 1: System diagram

bag control module to automatically detect a collision. It has a manual button to request help for a passenger with health problems or to report an accident of another vehicle. It has a GPS receiver, for locating the vehicle and its immediate path just before the accident.

The United Kingdom has an existing service, called the Public Service Answering Point (PSAP), these already exist and are operated by BT999 and Cable \& Wireless which uses different switches, databases and call handling software [4]. The eCall unit automatically contacts the PSAP. They then pass information to the many emergency service control rooms dispatching emergency services. The device has a data channel to send out a message about the vehicle and its location to PSAP. It also consists of a voice channel for operators to talk to those in the vehicle, to gain more detail and reassure the occupants [5].

Contran 245 is legislation in Brazil, requiring all new vehicles produced in, or imported to Brazil be equipped with GPRS-enabled tracking modules to reduce vehicle theft. This project is currently on track and in the implementation phase [5]. This legislation could make Brazil one of the largest telematics markets in the world, both for basic vehicle tracking and extended services, ranging from basic tracking required by the legislation to services like fleet management and behavior modeling for insurance purposes.

In the United States, although not a current law, many insurance companies are beginning to implement usage-based insurance to lower insurance prices for customers, as well as promote safe driving.

In Italy the benefits of usage-based insurances are already leading to a new law which is at the moment under parliament approval [2].

In Africa it is already an industry standard that high-end motor vehicles have GPS tracking devices installed in to be insured. Many insurance companies support and use a usage-based system to determine risk factors. It is centered on measurable information like the odometer reading of the vehicle number of minutes the vehicle is being used, speed and time-of-day information in addition to distance or time traveled. Other data could include your location and driving behavior such as speeding, excessive braking [6].

In Kwazulu-Natal, a province in South Africa, approximately 3000 minibus taxis will be equipped in the next two years with state-of-the-art electronic fare collection sys-

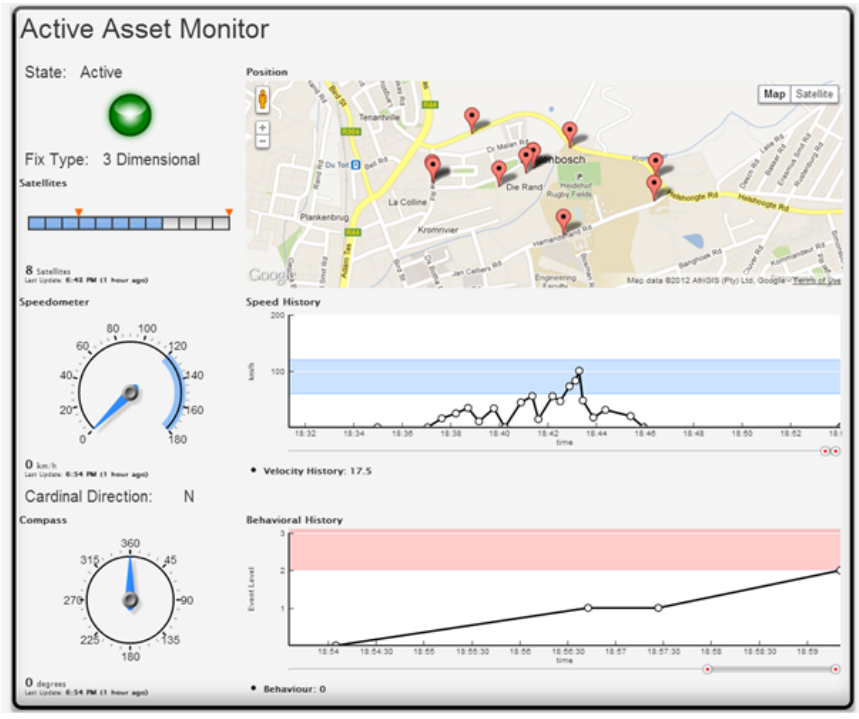

Fig. 2: SMART platform

tems called TAP-I-FARE. The uMgungundlovu Regional Taxi Council in Kwazulu-Natal, with its 40 Taxi Associations, collectively owns 3700 taxis and transports more than 500 000 people daily [7]. The existing payment system is cashbased which results in checks and balances regarding vehicle condition, driver behavior and passenger safety very difficult to manage, monitor and enforce.

Safety is a key selling point for the automotive industry, which are fitting systems that either actively reduce the chances of a crash or passively mitigate its effects. The challenge is to transform raw data into useful information for customers and especially to create new services from this enhanced information.

\section{System Design}

The system is illustrated in Fig. 1. The input division illustrates that all the raw data is acquired from an accelerometer and a GPS device. The processing division illustrates that only selected data is filtered and then the micro-controller determines which data to output where. The output division illustrates the three types of outputs this device facilitates. Mass data is logged on secondary storage, critical states are indicated on LEDs (Light Emitting Diode) on the device itself, and data is sent via a modem to the web-server for further interpretation and processing. The channel division illustrates that to transfer data from the modem to the webserver facilitates the available GSM networks as channel(s) for communication. The user interface division illustrates that the web-server processes and finalizes the data to be viewed and interpreted by the end-user.

Accelerometers has a wide range of features including sensing of acceleration, tilt, rotation, shock, vibration and multiple DOF (Degrees-of-Freedom). The application of an accelerometer in this project is primarily to sense acceleration and secondarily, tilt. 
Static and dynamic acceleration measurements are two different applications of the accelerometer. Static acceleration includes the measurement of the earth's gravity as the device is rotated into various positions, and is used to measure the tilt angle of the object it is attached to.

Dynamic acceleration is the measurement of the physical acceleration vector of an object only considering the difference in acceleration. The accelerometer is still subject to the gravitational force of the environment, but this information is disregarded in the process of obtaining multi-axial acceleration.

Whenever certain thresholds are surpassed the microcontroller will inform the web-server of different events. These events will be logged and a history can be viewed indicating the exact time and severity of the offense. The first level of reckless behavior will be triggered when the velocity of the vehicle exceeds $100 \mathrm{~km} / \mathrm{h}$, which is the legal speed limit for minibus taxis in South Africa.

The second level of reckless behavior will be triggered when the velocity of the vehicle exceeds $120 \mathrm{~km} / \mathrm{h}$, or a reading exceeding the predetermined thresholds $\left(X_{T} \& Y_{T}\right)$ on the perpendicular axes of the accelerometer. These thresholds were derived experimentally.

The third level of reckless behavior will be triggered when the vehicle exceeds $140 \mathrm{~km} / \mathrm{h}$, or when the acceleration thresholds $\left(X_{T} \& Y_{T}\right)$ exceeds their predetermined values by $20 \%$. This event will also send an email to the asset's owner or coordinator informing them of this occurrence.

The last event is sent when fatal parameters are measured. Parameters are considered fatal if the accelerometer measures a vehicle tilt angel of more than 45 on any perpendicular axis or an acceleration measurement of $90 \%$ the maximum range of the accelerometer. Only $11.13 \%$ of the maximum range of the accelerometer is used to determine reckless driving. Severe accidents results in accelerations above the maximum range of the accelerometer. Thus a reading within the top 10 percentile of the accelerometer range will indicate a collision of some sort.

\section{A. Calculating Tilt}

When considering raw data sampled at $f_{s}=20 \mathrm{~Hz}$ with a resolution of $275 \mathrm{mV} / \mathrm{g}$ one can calculate the tilt angle of a vehicle also known as the roll $(\alpha)$ and pitch $(\beta)$. These are the angles between the vehicle's perceptive $\mathrm{X}$-axis or Yaxis and the stationary $\mathrm{Z}$-axis respectively as illustrated in Figure 3. The accelerometer does not measure direction like a compass, thus we are not interested in the yaw, the output on the Z-axis. Information regarding the direction in which the vehicle is traveling will be obtained from the GPS. Tilt is a static measurement where gravity is the acceleration being measured. Therefore, to achieve the highest degree resolution of a tilt measurement, a low-g, high-sensitivity accelerometer is required. The typical output of capacitive, micro-machined accelerometers is nonlinear and is best described by a sine function [14]. Equation 2 is a derivation to calculate the roll $(\alpha)$ and pitch $(\beta)$ respectively. $v_{o} u t$ is the accelerometer

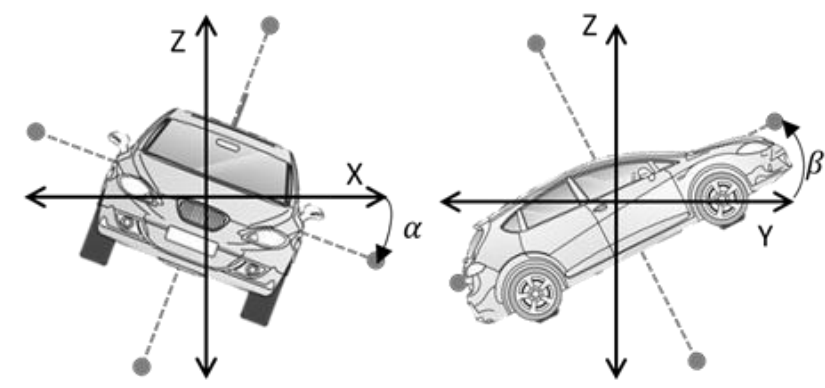

Fig. 3: Calculating tilt with static acceleration.

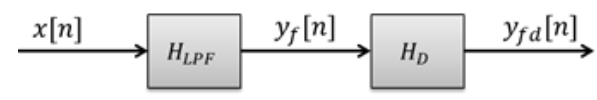

Fig. 4: Low pass filter and differentiator used with dynamic acceleration

output in volts, $v_{o} f f$ set $=1650 \mathrm{mV}$ is the $0 \mathrm{~g}$ offset voltage, $\Delta v / \Delta g=275 \mathrm{mV} / \mathrm{g}$ is the resolution, $1 g=9.80665 \mathrm{~m} / \mathrm{s}^{2}$ is the earth's gravity and $\theta$ is the angle of tilt.

$$
\begin{gathered}
v_{\text {out }}=v_{\text {off set }}+\frac{\Delta v}{\Delta g} g \sin \theta \\
\theta=\arcsin \left(\frac{v_{\text {out }}-v_{\text {offset }}}{\frac{\Delta v}{\Delta g}}\right)
\end{gathered}
$$

\section{B. Calculating Acceleration}

In order to measure dynamic acceleration the difference in acceleration must be obtained. It must also be considered that a vehicle can experience tangential (forward) or normal (sideways) acceleration while on a slope, i.e. the tilt (and gravitational effects) must not influence the dynamic acceleration measurement, which will use absolute values. The $\mathrm{Z}$-axis acceleration is also monitored for extreme values but reckless driving behavior will primarily be detected from acceleration vector.

In order to retrieve the acceleration vector with a zero offset, the raw data must be differentiated. Figure 4 shows the LPF with a differentiator. A finite-difference approximation was derived to approximate a differentiator in (5). The approximation becomes more accurate as $f_{s}$ increases.

$$
\begin{aligned}
& {\frac{d y_{f}(t)}{d t}}_{t=n T_{s}}=y_{f d}[n]=\frac{y_{f}[n]-y_{f}[n-1]}{T_{s}} \\
& Z\left\{{\frac{d y_{f}(t)}{d t}}_{t=n T_{s}}\right\}=Z\left\{y_{f d}[n]\right\}=\frac{1}{t_{s}} Y_{f}(z)-z-1 Y_{f}(z) \\
& H_{D}(z)=\frac{Y_{f d}(z)}{Y_{f}(z)}=\frac{1-z-1}{T_{s}}=f_{s}\left(1-z^{-1}\right)
\end{aligned}
$$




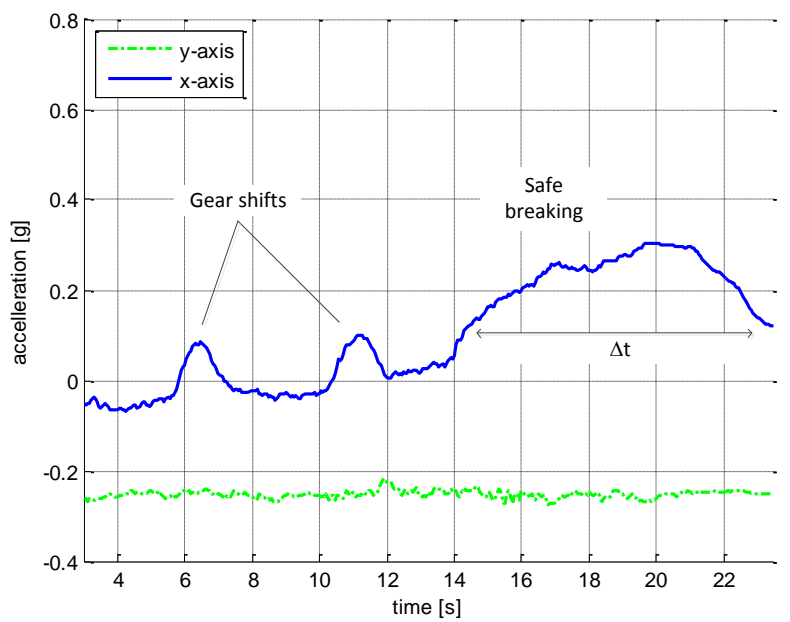

(a) Braking - safe.

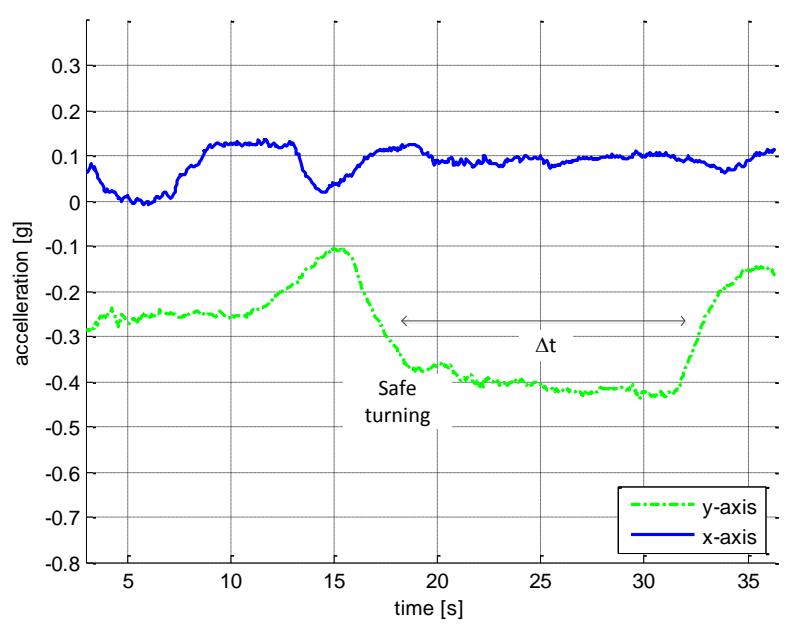

(c) Traffic circle - safe.

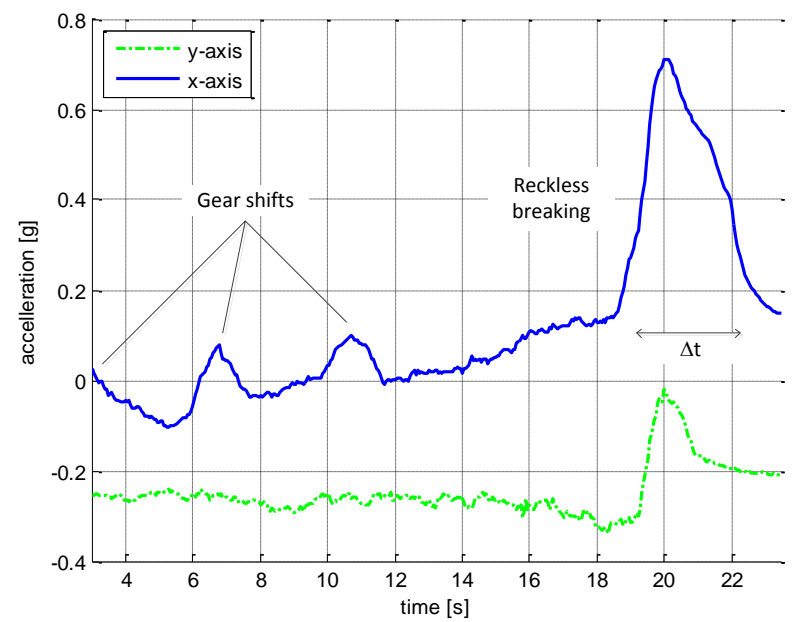

(b) Braking - reckless.

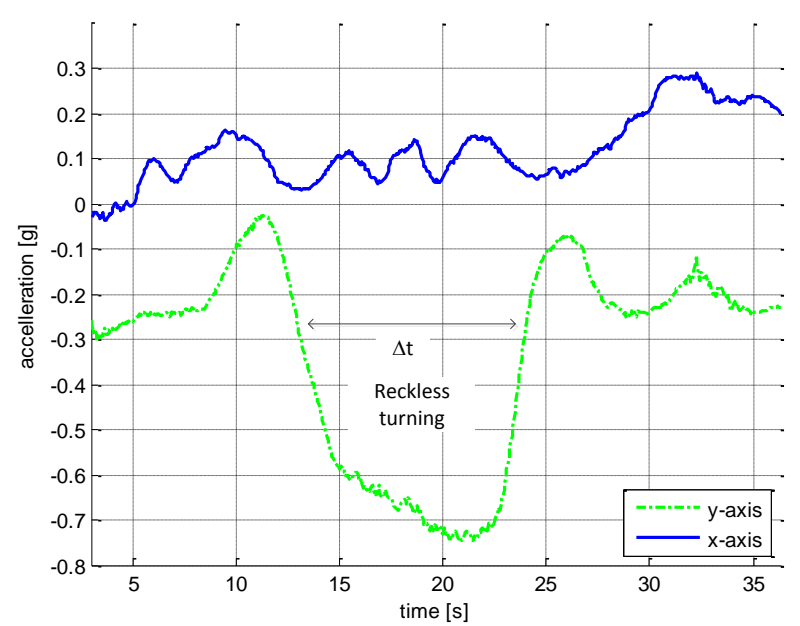

(d) Traffic circle - reckless.

Fig. 5: Performance results.

\section{RESULTS}

The results presented in this section describes the results of the exploration tests, performed to ensure that the accelerometer measurements can in fact distinguish between reckless and safe driving. Two scenarios were tested: braking and turning. The strategy was to record accelerometer data while conducting a series of controlled tests.

The vehicle used was a manual front wheel drive with no ABS (Anti-lock Braking System). The device was set to record the accelerometer data continuously. Every recording includes the departure and stopping before and after each test.

\section{A. Accelerometer braking}

A quiet straight asphalt motorway was used. The vehicle was driven to obtain a speed of $60 \mathrm{~km} / \mathrm{h}$ before completely stopping. The tests were conveyed in two sections. Three tests were done by stopping in a regular and comfortable fashion after which an additional three tests were done by stopping in a reckless and uncomfortable manner. The objective is to obtain a threshold value that distinguishes between acceptable and reckless braking behavior.

1) Hypothesis: It was expected to be able to identify acceleration and deceleration patterns from the readings of the Y-axis. Furthermore it was expected to be able to differentiate between severities of acceleration and deceleration by observing the difference in the peak heights and offset values, and the duration the acceleration or deceleration occurred. In these tests the $\mathrm{X}$-axis should not have any significant meanings.

2) Results: Figures $5 \mathrm{a}$ and $5 \mathrm{~b}$ illustrate the results of the braking tests. In order to ease comparisons the vertical scale on all the figures were selected to range from 470 bits to 580 bits. Analyzing the results, it is clear in all the graphs where a gear was shifted. These graphs confirm that braking occurred while the vehicle was in third-gear. The results illustrate that normal braking results in a gradual and longer stopping time whereas reckless braking results in a shorter and higher deceleration. 
A threshold of $Y_{\text {Threshold }}, Y_{T}=40$ was implemented in the micro-controller to trigger on the events described in the system design. With the threshold set, the device reported reckless braking to the online platform, as expected, thus rendering this experiment successful.

\section{B. Accelerometer turning}

A large traffic circle was used for the turning tests. Every recording includes the departure and stopping before and after each test. The tests were conveyed in two sections. All the tests were done by entering and leaving the circle at the same node. Three tests were recorded in a regular and comfortable fashion after which an additional three tests were done in a reckless and uncomfortable manner. The objective is to obtain a threshold value that distinguishes between acceptable and reckless turning behavior.

1) Hypothesis: It was expected to be able to identify the normal component of the acceleration vector and also to identify patterns from the readings of the X-axis. Furthermore it was expected to be able to differentiate between severities of the normal acceleration by observing the difference in the peak heights and offset values, and the duration the acceleration or deceleration occurred. In these tests the Y-axis should not have any significant meanings.

2) Results: Figures 5c and 5d illustrate the results of the turning tests. Figure $5 \mathrm{c}$ illustrates the characteristics of entering, completing and exiting a circle. The results show a clear distinction between normal and reckless turning. It can be seen that the duration spent in the circle clearly differs from normal to reckless, a higher normal component of acceleration is expected when the velocity throughout the experiment is higher thus the circle is completed quicker. The results show that a left or right turn can be identified as the acceleration deviates from the $\mathrm{X}$-axis offset.

A threshold of $X_{\text {Threshold }}=X_{T}=50$ was implemented in the micro-controller to trigger on the events described in the system design. With the threshold set, the device reported reckless turns to the online platform, as expected, thus rendering this experiment successful.

\section{CONCLUSION}

In this work, a unit was developed to monitor a vehicle's location, velocity and driver behavior whilst transmitting relevant data to a centralized web-server. It was concluded from the literature survey that similar solutions that offer live tracking and driver behavior monitoring do not use accelerometers to monitor driver behavior - Only velocity and position is monitored to identify reckless behavior and compliance to operating zones. Data such as acceleration, speed and tilt angle of the vehicle was measured and used to characterize driver behavior. Accelerometers are very sensitive to vibrations and data captured from them was analyzed with caution. Through the use of various carefully designed LTI (Linear Time-Invariant) filters it was possible to detect various levels of reckless driving behavior from monitoring and interpreting multi directional acceleration. The difference between acceptable and reckless driving behavior is based on the occupants experience. Different people might have different experiences and will rate certain events differently. It was a challenge to quantify feelings and to create a model to respond to them. The measurements and simulations conducted gave insight to what aspects of driver behavior can be monitored and characterized with an accelerometer. The numerical thresholds obtained to distinguish between acceptable and reckless driving where implemented and proved to provide accurate reporting. Future work will include other scenarios of reckless driving patterns. We also intend to incorporate further statistical evaluation to replace the subjective classification of recklessness.

\section{ACKNOWLEDGMENT}

This work was financially supported by Trinity Telecoms South Africa and MTN South Africa.

\section{REFERENCES}

[1] Booysen MJ, "The relevance of Intelligent transportation Systems in subSaharan Africa", Development Bank of Southern Africa eDijest, pp. 20 - 23, online: http://goo.gl/tLngG, July 2012.

[2] S. Olariu and M. C. Weigle, Vehicular Networks from Theory to Practice, COMPUTER and INFORMATION SCIENCE SERIES, 2009.

[3] W. Wolf, Computers as Components: Principles of Embedded Computing System Design, Morgan Kaufmann, 2008.

[4] B. Principi, Legislation largest driver for telematics, Telecommuncations Online \& Horizon House Publications, 2012.

[5] J. Medland, UK Emergency Calls, British Telecommunications 999, 2011.

[6] D. McClure and A. Graham, eCall The Case for Deployment, The Department for Transport, 2006.

[7] J. Stojaspal, Telematics opportunties in Brazil and LATAM, part II, London: Telematics Update, 2012.

[8] Arrive Alive, Vehicle and Insurance Telematics, 202. [Online]. Available: www.arrivealive.co.za. [Accessed 1 October 2012].

[9] B. Zondi, $3000 \mathrm{KZN}$ Taxis to be fitted with smart card tech, on-board cameras, Engineering News, Creamer Media, p. 6, 13-19 July 2012.

[10] Atmel Corporation, Datasheet: ATmega640/1280/1281/2560/2561, Atmel Corporation, San Jose, USA, 2012.

[11] Freescale Semiconductor, Datasheet: 1.5g, 6g Three Axis Low-g Micromachined Accelerometer, Freescale Semiconductor, Tempe, Arizona, 2008.

[12] D. G. Manolakis and J. G. Proakis, Digital Signal Processing, in Principles, Algorithms, and Applications Fourth Edition, Upper Saddle River, New Jersey, Pearson Education, Inc., 2007, pp. 354-410.

[13] Road Safety Authority, Road Safety Authority, Transport Research Laboratory, UK, 2007. Online: http://www.rulesoftheroad.ie. [Accessed 15 September 2012].

[14] J. L. Meriam and L. G. Kraige, Engineering Mechanics Dynamics, in Engineering Mechanics Dynamics Sixth Edition, Virginia, John Wiley \& Son, Inc., 2008, pp. 21-26.

[15] D. Kecman, J. Lenard and P. Thomas, Safety of Seats in Minibuses Proposal for a Dynamic Test, Cranfield Impact Centre Ltd \& Vehicle Safety Research Centre, United Kingdon. 